\title{
ADAPTIVE NESTED RARE EVENT SIMULATION ALGORITHMS
}

\author{
Anand N. Vidyashankar \\ Department of Statistics \\ Volgeneau School of Engineering \\ Georgia Mason University \\ Fairfax, VA 22030, USA
}

\author{
Jie $\mathrm{Xu}$ \\ Systems Engineering \& Operations Research \\ Volgeneau School of Engineering \\ Georgia Mason University \\ Fairfax, VA 22030, USA
}

\begin{abstract}
Nested simulation algorithms are used in several scientific investigations such as climate, statistical mechanics, and financial and actuarial risk management. Recently, these methods have also been used in the context of Bayesian computations and are referred to as Nested Sampling. In several of these problems, the inner level computation typically involves simulating events with very small probability, leading to rare event importance sampling methods. The quality of the resulting estimates depend on the allocation of computational resources between inner and outer level simulations. We introduce a novel adaptive rare event simulation algorithm that allocates the computational resources by taking in to account marginal changes in the rare event probabilities. We establish the consistency and efficiency of our algorithm and theoretically and numerically compare our results with the non-adaptive methods. We illustrate the proposed methods with several examples.
\end{abstract}

\section{INTRODUCTION}

Multilevel models such as hierarchical or random effects models are useful to describe complex scientific systems that also incorporate multiple sources of uncertainty. In these models, closed form expressions for point and interval estimates of the parameters are usually unavailable and one frequently has to resort to Monte Carlo (MC) methods. In typical situations, these MC methods also involve multiple levels of simulation and "optimal" allocation of computational resources amongst the levels of simulations is necessary for an accurate assessment of the variability. Recently, these issues have received intense attention in financial risk management (Gordy and Juneja 2010; Liu and Staum 2010; Sun, Apley, and Staum 2011).

Understanding of rare but high-risk events is critical to planning for alternative modes of action when such events arise. It is frequently impossible, if not illegal, and expensive to conduct experiments that yield rare events. At this time, simulation is perhaps the only strategy available to assess and quantify the probabilities of rare events. However since multiple, typically unknown, factors can lead to rare events, a hierarchical modeling idea can be a beneficial approach to identify and evaluate the worst-case scenarios. This approach is especially useful in problems involving risk management where planning appropriate mitigation strategies based on simulation results and historical data can reduce the consequences of these high-risk events when they occur.

Standard MC methods for simulating rare event probabilities are not applicable since variance of the estimator tends to be larger than the estimated probability. Variance reduction techniques such as importance sampling and multilevel splitting are typically used to address the inadequacy of the standard MC estimators of the tail probability. This paper is concerned with the adaptive nested rare event simulation algorithm where the simulation algorithm invokes the sampling distribution so as to simulate random variables in the part of the sample space that has significant effect on the calculations. To provide a unified focus that 


\section{Vidyashankar and $\mathrm{Xu}$}

includes several useful examples, we focus on a class of stochastic fixed point equations (SFPE) referred to as Letac's model E that incorporate unobservable latent effects. In this context, we consider an algorithmic solution to the problem of adaptive estimation of the rare event probabilities.

The rest of the paper is organized as follows: Section 2 introduces stochastic fixed point equations and provides several examples while Section 2.1 is concerned with Stochastic fixed point equations with random effects. Section 3 deals with nested rare event simulation algorithm for the estimation of the rare event probability while Section 3.1 is concerned with allocation of resources. Section 4 contains some numerical examples and a brief discussion.

\section{STOCHASTIC FIXED POINT EQUATIONS}

A general stochastic fixed point equation has the form

$$
V \stackrel{\mathscr{D}}{=} f(V)
$$

where $f$ is a random function satisfying certain regularity conditions and is independent of $V$. When $f(v)=A v+B$, where $(A, B)$ is independent of $V$ and $\mathbf{E}[\log A]<0$, this problem has a long history starting with the works of Solomon (1972), Kesten (1973), Vervaat (1979), and Letac (1986). Suppose that we begin with the SFPE (2.1) and assume that this equation can be written as a functional equation of the form

$$
V \stackrel{\mathscr{D}}{=} F(V, Y)
$$

where $F: \mathbb{R} \times \mathbb{R}^{d} \rightarrow \mathbb{R}$ is assumed to be deterministic, measurable, and continuous in its first component. For convenience of notation, we will often write $F_{y}(v)=F(v, y)$, where $y \in \mathbb{R}^{d}$.

Let $v$ be an element of the range of $F$, and let $\left\{Y_{n}\right\}$ be an i.i.d. sequence of random variables such that $Y_{i} \stackrel{\mathscr{D}}{=} Y$ for all $i$. Then the forward equation generated by the SFPE (2.2) is defined by

$$
V_{n}(v)=F_{Y_{n}} \circ F_{Y_{n-1}} \circ \cdots \circ F_{Y_{1}}(v), \quad n=1,2, \ldots, \quad V_{0}=v,
$$

while the backward equation generated by this SFPE is defined by

$$
Z_{n}(v)=F_{Y_{1}} \circ F_{Y_{2}} \circ \cdots \circ F_{Y_{n}}(v), \quad n=1,2, \ldots, \quad Z_{0}=v .
$$

Note that the backward recursion need not be Markovian, but for every $v$ and every integer $n, V_{n}(v)$ and $Z_{n}(v)$ are identically distributed. The Letac's principle states that when the backward recursion converges to a random variable $Z$ almost surely (a.s.) and is independent of its starting value $v$, then the stationary distribution of $\left\{V_{n}\right\}$ is the same as the distribution of $Z$. This is precisely described in the following lemma (Letac 1986).

Lemma 2.1. Let $F: \mathbb{R} \times \mathbb{R}^{d} \rightarrow \mathbb{R}$ be deterministic, measurable, and continuous in its first component; that is, $F_{Y}$ is continuous, where $Y: \mathbb{R}^{d} \rightarrow \mathbb{R}$ for some $d \in \mathbb{Z}_{+}$. Suppose that $\lim _{n \rightarrow \infty} Z_{n}(v):=Z$ exists a.s. and is independent of its initial state $v$. Then $Z$ is the unique solution to the SFPE (2.2). Furthermore, $V_{n}(v) \Rightarrow V$ as $n \rightarrow \infty$, where $V$ is independent of $v$, and the law of $V$ is same as the law of $Z$.

Starting from the general SFPE (2.2), we generate the forward recursive sequence (2.3) and study the evolution of this process. We will focus on the quasi-linear recursion, referred to as Letac's Model E,

$$
V \stackrel{\mathscr{D}}{=} F_{Y}(V), \text { where } F_{Y}(v)=A \max (v, D)+B,
$$

and $Y=(A, B, D)$, which generates the forward recursive sequence

$$
V_{n}=A_{n} \max \left(V_{n-1}, D_{n}\right)+B_{n}, \quad n=1,2, \ldots,
$$


with i.i.d. driving sequence $\left\{\left(A_{n}, B_{n}, D_{n}\right): n=1,2, \ldots\right\}$. Iterating this forward recursion, we obtain that

$$
V_{n}=\max \left(\sum_{i=0}^{n} B_{i} \prod_{j=i+1}^{n} A_{j}, \bigvee_{k=1}^{n}\left[\sum_{i=k}^{n} B_{i} \prod_{j=i+1}^{n} A_{j}+D_{k} \prod_{j=k}^{n} A_{j}\right]\right)
$$

Iterating the corresponding backward recursion and setting $B_{i}^{*}=B_{i}$ for $i=1, \ldots, n$ and $B_{n+1}^{*}=V_{0}$, we obtain that

$$
\tilde{Z}_{n}=\max \left(\sum_{i=1}^{n+1} B_{i}^{*} \prod_{j=1}^{i-1} A_{j}, \bigvee_{k=1}^{n}\left[\sum_{i=1}^{k} B_{i} \prod_{j=1}^{i-1} A_{j}+D_{k} \prod_{j=1}^{k} A_{j}\right]\right)
$$

Under additional assumptions, the forward recursive sequence is a Markov chain possessing a stationary distribution and a regenerative structure which we describe below.

For the remainder of this section, let $\left\{V_{n}\right\}$ denote a Markov chain on a general state space ( $\left.\mathbb{S}, \mathscr{S}\right)$, and assume that this chain is aperiodic, countably generated, and irreducible with respect to its maximal irreducibility measure, which we will denote by $\varphi$. Let $P$ denote the transition kernel of $\left\{V_{n}\right\}$. From Section 2.1 onwards, we will take $\left\{V_{n}\right\}$ to be the Markov chain generated by a forward recursion, but for the remainder of this section we will assume it to be an arbitrary chain.

It is known that if $\left\{V_{n}\right\}$ is aperiodic and $\varphi$-irreducible, then it satisfies a minorization, namely

$$
\delta \mathbf{1}_{\mathscr{C}}(x) v(E) \leq P^{k}(x, E), \quad x \in \mathbb{S}, \quad E \in \mathscr{S}, \quad k \in \mathbb{Z}_{+},
$$

for some set $\mathscr{C}$ with $\varphi(\mathscr{C})>0$, some constant $\delta>0$, and some probability measure $v$ on $(\mathscr{S}, \mathbb{S})$. The set $\mathscr{C}$ is called a "small set" or " $\mathscr{C}$-set." It is no loss of generality to assume that this $\mathscr{C}$-set is bounded, and we will make such an assumption throughout this article.

An important consequence of $(\mathscr{M})$, first established in Athreya and Ney (1978) and Nummelin (1978), is that it provides a regeneration structure for the chain; see Nummelin (1984), Theorem 3.2. Letting $\tau$ denote a typical regeneration time, and letting

$$
N_{u}=\sum_{n=0}^{\tau-1} \mathbf{1}_{(u, \infty)}\left(V_{n}\right),
$$

where $V_{0} \sim v$, the following representation formula is obtained from Athreya-Ney-Nummelin regeneration lemma (cf. Nummelin (1984), p. 75).

Lemma 2.2. Assume that $(\mathscr{M})$ holds with $k=1$. Then for any $u \in \mathbb{R}$,

$$
\mathbf{P}(V>u)=\frac{\mathbf{E}\left[N_{u}\right]}{\mathbf{E}[\tau]} .
$$

We now turn to a few applications of the quasi-linear recursion (2).

Example 2.1 Consider an insurance company which earns capital according to a discrete-time CramérLundberg model, but which also invests its surplus capital, earning stochastic interest $\left\{R_{n}: n=1,2, \ldots\right\}$. Assume $\left\{R_{n}\right\}$ is i.i.d. and set $A_{n}=R_{n}^{-1}$ for all $n$. Let $L_{n}$ denotes the losses from the insurance business at time $n$. Then the cumulative discounted losses of the company at time $n$ are given by

$$
\mathscr{L}_{n}=A_{1} L_{1}+\left(A_{1} A_{2}\right) L_{2}+\cdots+\left(A_{1} \cdots A_{n}\right) L_{n},
$$

and it can be shown that ruin occurs if and only if $\mathscr{L}:=\left(\sup _{n \in \mathbb{Z}_{+}} \mathscr{L}_{n}\right) \vee 0$ exceeds a positive barrier at level $u$, where $u$ is the company's initial capital; $c f$. Collamore (2009), Section 3. By observing that $\mathscr{L}=A_{1} L_{1}+A_{1} \max \left\{0, \sup _{n \in \mathbb{Z}_{+}} \mathscr{L}_{n}^{(1)}\right\}$, where $\mathscr{L}_{n}^{(1)}:=\sum_{i=2}^{n+1}\left(A_{2} \cdots A_{i}\right) L_{i}$, we see that $\mathscr{L}$ satisfies the SFPE

$$
\mathscr{L} \stackrel{\mathscr{D}}{=}(A \mathscr{L}+B)^{+}, \text {where }(A, B) \stackrel{\mathscr{D}}{=}\left(A_{1}, A_{1} L_{1}\right) .
$$




\section{Vidyashankar and $\mathrm{Xu}$}

Example 2.2 The well-known $\operatorname{ARCH}(1)$ and $\operatorname{GARCH}(1,1)$ financial time series models satisfy the linear recursion $f(v)=A v+B$, where $A$ and $B$ are positive random variables. It is worth noting that in the $\operatorname{ARCH}(1)$ case, the pair $(A, B)$ is dependent. For a description of ARCH and GARCH processes, see Embrechts et al. (1997), Mikosch (2003), or Section 3 of Collamore (2009).

Example 2.3 In a life-insurance context, one often studies the future discounted losses of the company, namely the perpetuity sequence, where $\left\{A_{n}\right\}$ denotes a stochastic discount factor and $\left\{L_{n}\right\}$ the future losses or obligations of the company. Then it is of interest to study the steady-state tail behavior of $\mathscr{L}_{\infty}:=\lim _{n \rightarrow \infty} \mathscr{L}_{n}$, and a simple argument yields that $\mathscr{L}_{\infty} \stackrel{\mathscr{D}}{=} A \mathscr{L}_{\infty}+B$. Thus we are back in the previous example.

The recursion (2) also includes multiplicative random walk (by taking $D=1$ and $B=0$ ) and branching processes in random environments. Indeed, when viewed from an applied perspective, the recursion (2) is actually seen to be quite general, including a surprisingly rich collection of examples which are of practical interest.

\subsection{Stochastic Fixed Point Equations incorporating Random Effects}

We now turn to the Letac's model E incorporating random effects; that is, SFPEs of the form

$$
V(\xi) \stackrel{d}{=} A(\xi) \max \{V(\xi), D(\xi)\}+B(\xi),
$$

where $\xi$ is to be thought of as representing the scenarios or unobserved latent effects. We make the simplifying assumption that $\xi$ is a discrete finitely supported random variable with state space $S=\left\{s_{1}, s_{2}, \cdots s_{k}\right\}$. Let $\mathbf{P}\left(\xi=s_{j}\right)=p(j), j=1,2, \cdots k$. We shall assume that $\mathbf{E}\left[\left(A\left(s_{j}\right)\right)^{\alpha(j)} \mid \xi=s_{j}\right]<1$ for a unique $\alpha(j)$ for all $j-1,2, \cdots k$. Our first theorem is an extension of the result of Collamore and Vidyashankar (2013) to the context of SFPEs incorporating random effects. We start with the following assumptions:

Hypotheses:

$\left(H_{1}\right)$ The random variable $A\left(s_{j}\right)$ has an absolutely continuous component for all values of $1 \leq j \leq k$ with respect to Lebesgue measure, and satisfies the equation

$\Lambda\left(\xi\left(s_{j}\right)\right)=0$ for some $\xi\left(s_{j}\right) \in(0, \infty) \cap \operatorname{dom}\left(\Lambda^{\prime}\right)$ for all $1 \leq j \leq k$.

$\left(H_{2}\right) \mathbf{E}\left[\left|B\left(s_{j}\right)\right|^{\xi\left(s_{j}\right)}\right]<\infty$ and $\mathbf{E}\left[\left(A\left(s_{j}\right)\left|D\left(s_{j}\right)\right|\right)^{\xi}\right]<\infty$, for all $1 \leq j \leq k$

$\left(H_{3}\right) \mathbf{P}\left(A\left(s_{j}\right)>1, B\left(s_{j}\right)>0\right)>0$ or $\mathbf{P}\left(A\left(s_{j}\right)>1, B\left(s_{j}\right) \geq 0, D\left(s_{j}\right)>0\right)>0$ for all $1 \leq j \leq k$.

Our main theorem concerns the asymptotic behavior of the rare event probability, averaged across the scenarios.

Theorem 2.1. Assume Letac's Model E, and suppose that $\left(H_{1}\right),\left(H_{2}\right)$, and $\left(H_{3}\right)$ are satisfied. Let $s_{k 0}=$ $\arg \min \xi\left(s_{j}\right)$ and $\xi\left(s_{k 0}\right)=\xi^{*}$. Then

$$
\lim _{u \rightarrow \infty} u^{\xi^{*}} \mathbf{P}(V(\xi)>u)=C
$$

for a finite positive constant $C$.

The quantity $P(V(\xi)>u)$ can be referred to as the marginal tail probability and the result shows that the rate of decay of the marginal tail probability is determined by those scenarios that lead to the slowest decay.

\section{NESTED ADAPTIVE RARE EVENT SIMULATION ALGORITHM}

As mentioned previously in the Section 2, the main idea behind the analysis is the realization that under certain moment and regularity conditions, forward iterations of the SFPE generates a positively recurrent geometrically ergodic Markov chain and hence an algorithm for estimating the tails can be based on the regeneration techniques. Recently, Collamore et al. (2012) used a novel approach involving a dual change 


\section{Vidyashankar and $\mathrm{Xu}$}

of measure over a random time interval simulated over the finite-time excursions emanating from a set $\mathscr{C}$ and returning to this set, to develop a rare event simulation algorithm for the Letac's model E. Our first step is to extend this algorithm in the presence of scenarios. To this end, we need to first relate the tail probability to the number of exceedances of $V(\xi)$, for every realized value $\xi$.

Since, conditioned on $\xi, V_{n}(\xi)$ is a stationary ergodic Markov chain, fix the C-set $\mathscr{C}=[-M, M]$; let $\pi(\xi)(\cdot)$ denote the stationary distribution and define the probability measure on the Borel subsets of $\mathscr{C}$ as:

$$
\gamma_{\xi}(E)=\frac{\pi_{\xi}(E)}{\pi_{\xi}(\mathscr{C})} .
$$

Define $K(\xi)=\inf \left\{n \geq 1: V_{n}(\xi) \in \mathscr{C}\right\}$ and $N_{u}(\xi)=\sum_{i=1}^{K(\xi)-1} I_{\left[V_{n}(\xi)>u\right]}$. Since $\xi$ is a finitely supported discrete random variable, one can show that

$$
\mathbf{P}(V(\xi)>u)=\sum_{j=1}^{k} \mathbf{P}\left(V\left(s_{j}\right)>u \mid \xi=s_{j}\right) p_{j}=\sum_{j=1}^{k} \mathbf{E}_{\gamma\left(s_{j}\right)}\left[N_{u}\left(s_{j}\right) \mid \xi=s_{j}\right] p_{j}
$$

Before we move on to describe the rare event simulation algorithm, let $\mu(\xi)$ denote the distribution of $(\log A, B, D)$ and $\mu_{\alpha(\xi)}$ as the $\alpha(\xi)$ shifted distribution

$$
\mu_{\alpha(\xi)}(E) \equiv \frac{1}{\lambda(\xi)(\alpha)} \int_{E} e^{\alpha(\xi) x} d \mu(\xi)(x, y, z), E \in \mathscr{B}\left(\mathbb{R}^{3}\right),
$$

where $\mathscr{B}\left(\mathbb{R}^{3}\right)$ denotes the Borel subsets of $R^{3}$, and $\lambda(\xi)(\alpha)=\mathbf{E}\left[A(\xi)^{\alpha(\xi)} \mid \xi\right]$. We notice here that the measures $\mu(\xi)$ and the shifted measure $\mu_{\alpha(\xi)}$ are well-defined conditioned on $\xi$. Our algorithm proceeds as follows: For every sceanrio $\xi$, generate the process $V_{n}(\xi)$ according to the shifted measure (which drifts to infinity) until it exceeds $u$ and reverts to the original measure after the exceedance. This measure depends on the scenario and hence is termed as adaptive dual measure. Let $\mathfrak{D}(\xi)$ denote the dual change of measure.

We now turn to describing in detail the steps of the nested rare event simulation algorithm for estimating the marginal tail probability $P(V(\xi)>u)$, where $V(\xi)$ is the stationary solution of (2.4). Let $T_{u}(\xi)$ denote the first time $V_{n}(\xi)$ exceeds $u$ and $K(\xi)$ the first time the process returns to the set $\mathscr{C}$ after exceeding $u$. This implies that for all realizations $K(\xi) \geq T_{u}(\xi)$. The algorithm proceeds as follows:

1. Generate the scenario sequence $\xi_{1}, \xi_{2}, \cdots \xi_{n}$.

2. Given the scenario $\xi$, estimate the stationary measure $\pi_{\xi}(\cdot)$ as follows: conditioned on $\xi$, generate i.i.d. realizations $\left\{V_{n, j}(\xi): j=1,2, \cdots r\right\}$ of $V_{n}(\xi)$ and estimate

$$
\hat{\pi}_{\xi, r}(\mathscr{C})=\frac{1}{r} \sum_{l=1}^{r} I_{\left[V_{n, l} \in \mathscr{C}\right]} .
$$

Set $\hat{\gamma}_{\xi, r}(E)=\frac{\hat{\pi}_{\xi}(E)}{\hat{\pi}_{\xi}(\mathscr{C})}$, for any Borel set $E \in \mathscr{C}$; set $\hat{\gamma}_{r}(\xi)\left(\mathscr{C}^{c}\right)=0$.

3. Generate the process with $V_{0}(\xi) \sim \gamma_{\xi, r}$. Now, given $V_{n-1}(\xi)$, generate $V_{n}(\xi)$ using the forward iteration and $\alpha(\xi)$ shifted distribution. If $V_{n}(\xi) \in \mathscr{C}$, then stop and set $\mathscr{E}_{u}(\xi)=0$. If $V_{n}(\xi)>u$, then goto step 4; else repeat Step 3.

4. Generate the process according to the original distribution until the first time the process enters $\mathscr{C}$.

5. Compute the importance sampling estimator $\mathscr{E}_{u}(\xi)=N_{u}(\xi) e^{-\alpha(\xi) S_{T_{u}}(\xi)} I_{\left[T_{u}(\xi)<K(\xi)\right]}$, where $N_{u}$ denotes the number of exceedances of the process $V_{n}(\xi)$ above $u$ prior to time $K(\xi)$.

6. Repeat the steps 3-5 $m$ times each time calculating the importance sampling (IS) estimator, yielding the the $j^{\text {th }}$ realization $\mathscr{E}_{u, j}(\xi)$. 
7. Compute the average $\hat{\mathscr{E}}_{u, m}(\xi)=\frac{1}{m} \sum_{j=1}^{m} \mathscr{E}_{u, j}(\xi)$ and set $\pi_{r, \xi} \hat{\mathscr{E}}_{u, m}(\xi)$ as the IS estimator of $\mathbf{P}(V(\xi)>$ $u \mid \xi)$.

8. Compute the average $\hat{\mathscr{E}}_{u}(m, n)=\frac{1}{n} \sum_{i=1}^{n} \hat{\mathscr{E}}_{u, m}\left(\xi_{i}\right)$ and set it as the IS estimator of $P(V(\xi)>u)$.

We emphasize here that $\hat{\mathscr{E}}_{u}(m, n)=\hat{\mathscr{E}}_{u}(m, n, r)$ since the initial distribution of the algorithm depends on $r$. Our second theorem describes the properties of the algorithm.

Theorem 3.1. Under the hypotheses $(\mathrm{HO})-(\mathrm{H} 3)$ the following holds:

$$
\lim _{n \rightarrow \infty} \lim _{r \rightarrow \infty} \lim _{m \rightarrow \infty} \hat{\mathscr{E}}_{u}(m, n, r)=\mathbf{P}(V(\xi)>u) .
$$

Furthermore, under the additional assumption that $B(\xi)>0$ and $D(\xi)>0$,

$$
\lim _{u \rightarrow \infty} \mathbf{E}_{\mathfrak{D}}\left[\frac{T_{u}(\xi)}{\log u} \mid T_{u}(\xi)<K(\xi)\right]=C_{1},
$$

where $C_{1}$ is a finite positive constant.

Using techniques from Collamore et al. (2012), it can be shown that the above algorithm has bounded relative error; that is,

$$
\sup _{u>0} u^{2 \alpha^{*}} \mathbf{E}\left[\mathscr{E}_{u}(m, n, r)^{2}\right]<\infty .
$$

\subsection{Allocation of Resources}

A natural next question concerns the allocation of budget between inner and outer simulations. We assume that the sampling rate is independent of the outcome. Let $c$ denote the total budget. Then $(m(c), n(c))$ represent the allocation of resources to inner and outer simulations respectively. Our next theorem describes the trade-off between the outer and inner simulation budget using the mean-square error criterion.

Theorem 3.2. The MSE of the IS estimator proposed by the above algorithm is minimized if $m(c) \sim c^{1 / 3} \log u$ as $u \rightarrow \infty$.

It is worthwhile to point out here that the budget $c$ depends on $u$. It follows from the above Theorem that $n(c) \sim c^{2 / 3}(\log u)^{-1}$. Thus, for large values of $u$, fewer number of scenarios will lead to increased variance of the estimator.

\section{IMPLEMENTATION}

A small simulation study detailing the trade-off assessed using the variances of the estimators is provided in Table 1. We simulated the ruin probability under the Cramér-Lundberg Model with Stochastic Investment described in Example 2.1. The initial capital $u$ is set to be between 1E5, 1E6, 1E7, and 1E8. We report $P(V>u)$, the MSE of the estimate, and the lower and upper bounds of the $95 \%$ confidence intervals. The number of claims $N(t)$ is a Poisson process with arrival rate 0.5. The total amount of claims in the interval $[0, t]$ is given by $\sum_{n=1}^{N_{t}} \zeta_{n}$, where $\left\{\zeta_{n}\right\}$ is i.i.d. and independent of $\left\{N_{t}\right\}$. We assume that $\left\{\zeta_{n}\right\}$ is exponentially distributed, and the mean of the exponential distribution has a $N(1,0.5)$ distribution with the negative part of the distribution truncated.

We use a total simulation budget $c=1,000,000$ and performed 200 macro replications to estimate the MSE of the nested rare event simulation estimator of the ruin probability $P(V>u)$ and the lower and upper bounds of the $95 \%$ confidence intervals. We should point out when we do not model the risk factors affecting the distribution of the mean loss size, and treat $\left\{\zeta_{n}\right\}$ as an exponential distribution with a mean of 1 (which is the approximately the mean of the truncated $N(1,0.5)$ distribution), the estimated ruin probability is much smaller. For example, when $u=1 \mathrm{E} 5, P(V>u)$ is estimated to be $1.98 \mathrm{E}-4$, much smaller than $3.40 E-4$. Therefore, without our nested rare event simulation methods, risk managers would considerably underestimate risks. 


\section{Vidyashankar and $\mathrm{Xu}$}

In Table 1, the third row of each experiment with a different $u$ uses an inner simulation budget computed by the formula $m(c)=c^{1 / 3} \log u$. We then experimented with $m(c) / 150, m(c) / 50,50 m(c), 500 m(c)$ and report the results in the other four rows for each experiment. From the results, we see that the ruin probability estimates $P(V>u)$ tend to be relatively stable for different choices of $m(c)$ (and thus $n(c)$ ), except for very small values of $n$, suggesting that the recommended choice of $m(c)$ is relatively robust in terms of the consistency of $P(V>u)$. The results also demonstrate that different choices of $m(c)$ can lead to different MSEs and confidence intervals, clearly demonstrating the importance of the outer-inner simulation budget allocation. The choice of $m(c)=c^{1 / 3} \log u$ seems to give relatively good MSEs, though not always the best. It is understandable since our initial result $m(c) c^{1 / 3} \log u$ involves an unknown constant term. But the small set of experiments confirms the importance to have enough scenarios, illustrated by the increase in MSE as $n(c)$ becomes smaller while holding the total budget $c$ constant.

Table 1: Nested Rare Event Simulation of the Ruin Probability under a Cramér-Lundberg Model with Stochastic Investment: $u$-initial capital, $m$-number of scenarios, $n$-number of inner simulation replications, LCL-lower bound of the $95 \%$ CI, UCL-upper bound of the $95 \%$ CI.

\begin{tabular}{|c|c|c|c|c|c|c|}
\hline$u$ & $n$ & $m$ & $P(V>u)$ & MSE & LCL & UCL \\
\hline $1 \mathrm{E} 5$ & 5 & 172650 & $3.50 \mathrm{E}-4$ & $4.03 \mathrm{E}-8$ & $8.67 \mathrm{E}-5$ & $8.65 \mathrm{E}-4$ \\
\hline $1 \mathrm{E} 5$ & 17 & 57550 & $3.36 \mathrm{E}-4$ & $1.38 \mathrm{E}-8$ & $1.57 \mathrm{E}-4$ & $6.14 \mathrm{E}-4$ \\
\hline $1 \mathrm{E} 5$ & 868 & 1151 & $3.40 \mathrm{E}-4$ & $7.29 \mathrm{E}-9$ & $1.97 \mathrm{E}-4$ & $5.14 \mathrm{E}-4$ \\
\hline $1 \mathrm{E} 5$ & 43478 & 23 & $3.42 \mathrm{E}-4$ & $7.23 \mathrm{E}-9$ & $2.03 \mathrm{E}-4$ & $5.06 \mathrm{E}-4$ \\
\hline $1 \mathrm{E} 5$ & 500000 & 2 & $3.38 \mathrm{E}-4$ & $6.66 \mathrm{E}-9$ & $2.01 \mathrm{E}-4$ & $5.01 \mathrm{E}-4$ \\
\hline $1 \mathrm{E} 6$ & 4 & 207150 & $7.84 \mathrm{E}-5$ & $1.69 \mathrm{E}-9$ & $2.51 \mathrm{E}-5$ & $1.69 \mathrm{E}-4$ \\
\hline 1E6 & 14 & 69050 & $8.54 \mathrm{E}-5$ & $1.05 \mathrm{E}-9$ & 4.09E-5 & $1.53 \mathrm{E}-4$ \\
\hline $1 \mathrm{E} 6$ & 724 & 1381 & $8.48 \mathrm{E}-5$ & $4.15 \mathrm{E}-10$ & 4.84E-5 & $1.28 \mathrm{E}-4$ \\
\hline $1 \mathrm{E} 6$ & 37037 & 27 & $8.83 \mathrm{E}-5$ & $4.72 \mathrm{E}-10$ & $5.20 \mathrm{E}-5$ & $1.29 \mathrm{E}-4$ \\
\hline $1 \mathrm{E} 6$ & 50000 & 2 & $8.51 \mathrm{E}-5$ & $4.30 \mathrm{E}-10$ & $4.85 \mathrm{E}-5$ & $1.22 \mathrm{E}-4$ \\
\hline $1 \mathrm{E} 7$ & 4 & 241650 & $3.44 \mathrm{E}-05$ & $1.13 \mathrm{E}-9$ & $3.87 \mathrm{E}-6$ & $1.14 \mathrm{E}-4$ \\
\hline $1 \mathrm{E} 7$ & 12 & 80850 & $3.50 \mathrm{E}-05$ & $2.92 \mathrm{E}-10$ & $1.30 \mathrm{E}-5$ & 7.36E-5 \\
\hline 1E7 & 620 & 1611 & $3.77 \mathrm{E}-05$ & $2.09 \mathrm{E}-10$ & $1.53 \mathrm{E}-5$ & $6.45 \mathrm{E}-5$ \\
\hline $1 \mathrm{E} 7$ & 31250 & 32 & $3.78 \mathrm{E}-05$ & $1.78 \mathrm{E}-10$ & $1.59 \mathrm{E}-5$ & $6.13 \mathrm{E}-5$ \\
\hline $1 \mathrm{E} 7$ & 333333 & 3 & $2.13 \mathrm{E}-05$ & $2.95 \mathrm{E}-10$ & $1.13 \mathrm{E}-5$ & $3.19 \mathrm{E}-5$ \\
\hline $1 \mathrm{E} 8$ & 3 & 276300 & $4.72 \mathrm{E}-6$ & $8.10 \mathrm{E}-12$ & $7.15 \mathrm{E}-7$ & $1.14 \mathrm{E}-5$ \\
\hline $1 \mathrm{E} 8$ & 10 & 92100 & $5.24 \mathrm{E}-6$ & $5.28 \mathrm{E}-12$ & $2.54 \mathrm{E}-6$ & $1.03 \mathrm{E}-5$ \\
\hline $1 \mathrm{E} 8$ & 542 & 1842 & $5.34 \mathrm{E}-6$ & $1.85 \mathrm{E}-12$ & $3.21 \mathrm{E}-6$ & $8.13 \mathrm{E}-6$ \\
\hline $1 \mathrm{E} 8$ & 27777 & 36 & $5.24 \mathrm{E}-6$ & $1.87 \mathrm{E}-12$ & $3.01 \mathrm{E}-6$ & 7.93E-6 \\
\hline $1 \mathrm{E} 8$ & 333333 & 3 & $5.46 \mathrm{E}-6$ & $1.78 \mathrm{E}-12$ & $3.11 \mathrm{E}-6$ & $9.10 \mathrm{E}-5$ \\
\hline
\end{tabular}

The overall simulation cost of the nested algorithm is a product of the cost for outer and inner simulations. Since the outer simulation costs are fixed (random draws from a probability distribution) and the inner simulation involves rare-event algorithm, the overall cost is determined by the running time of the inner simulation algorithm. This is described in Figure 1. We see that the importance sampling algorithm scales up very well to the increase in initial capital $u$, as suggested by the linear relationship between $\log u$ and the average number of SFPE iterations used by the inner level simulation importance sampling algorithm to generate a sample path leading to ruin. 


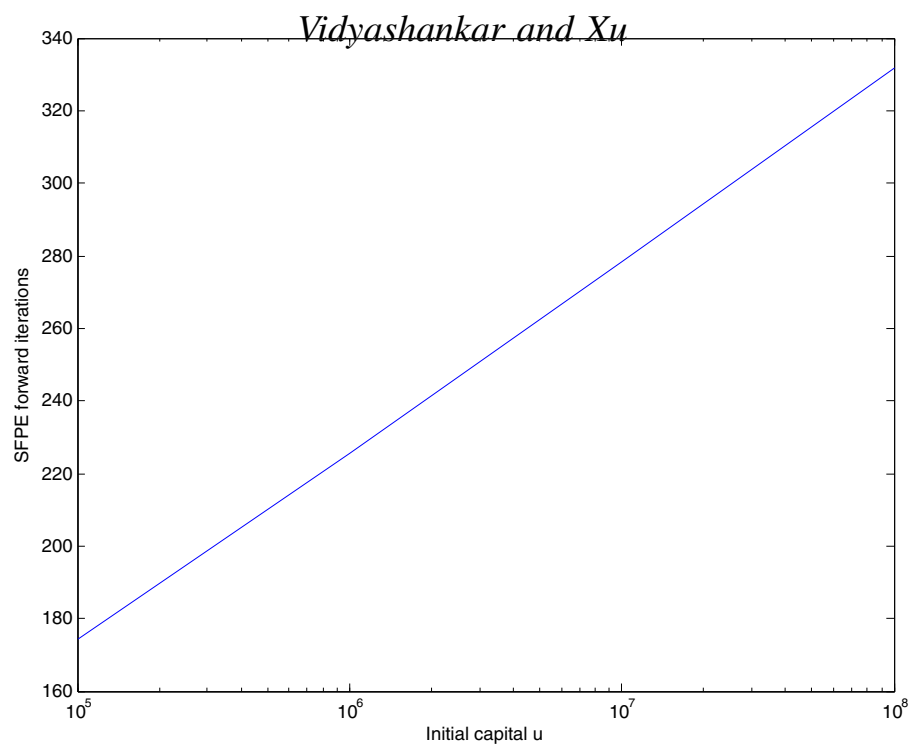

Figure 1: Number of SFPE forward iterations for importance sampling paths exceeding $u$.

\section{REFERENCES}

Athreya, K., and P. Ney. 1978. "A new approach to the limit theory of recurrent Markov chains". Trans. Amer. Math. Soc. 245:493-501.

Collamore, Jeffrey, F., G. Diao, and A. N. Vidyashankar. 2012. "Rare Event Simulations for processes Generated via Stochastic Fixed Point Equations". Submitted.

Collamore, Jeffrey, F., and A. N. Vidyashankar. 2013. "Tail Estimates for Stochastic Fixed Point Equations via Non-linear Renewas theory". Stochastic Processes and their Applications 123:3378-3429.

Collamore, J. F. 2009. "Random recurrence equations and ruin in a Markov-dependent stochastic economic environment". Ann. Appl. Probab. 19:1404-1458.

Embrechts, P., C. Klüppelberg, and T. Mikosch. 1997. Modelling Extremal Events for Finance and Insurance. Heidelberg: Springer-Verlag.

Kesten, H. 1973. "Random difference equations and renewal theory for products of random matrices". Acta Math. 131:207-248.

Letac, G. 1986. "A contraction principle for certain Markov chains and its applications. Random matrices and their Applications". Proceedings of AMS-IMS-SIAM Joint Summer Research Conference 1984. Contemporary Mathematics 50:263-273.

Mikosch, T. 2003. "Modeling dependence and tails of financial time series". In Extreme Values in Finance, Telecommunications, and the Environment, edited by B. Finkenstädt and H. Rootzén, 185-286. Boca Raton: Chapman and Hall.

Nummelin, E. 1978. "A splitting technique for Harris recurrent Markov chains". Z. Wahrsch. Verw. Gebiete 43:309-318.

Nummelin, E. 1984. General Irreducible Markov Chains and Non-negative Operators. Cambridge: Cambridge University Press.

Solomon, F. 1972. Random Walks in Random Environment. Ph.D. dissertation, Cornell University.

Vervaat, W. 1979. "On a stochastic difference equation and a representation of non-negative infinitely divisible random variables". Adv. Appl. Prob. 11:750-783.

\section{AUTHOR BIOGRAPHIES}

ANAND N. VIDYASHANKAR is an Associate Professor in the Department of Statistics at George Mason University. He received his Ph.D. in Statistics and Mathematics from Iowa State University and has held subsequent positions in the Departments of Statistics at the University of Georgia and Cornell University. 


\section{Vidyashankar and $\mathrm{Xu}$}

His main interests are in the areas of Branching Processes and Branching Random Walks, Stochastic Fixed Point Equations, Rare Event Simulations, Nested Simulations, Network Analysis, High-dimensional Statistical Inference, Robust Statistical Methods, and Risk Theory. He is a member of American Statistical Association, Institute of Mathematical statistics, and INFORMS. His email address is avidyash@gmu.edu and his web page is http://mason.gmu.edu/ avidyash/.

JIE XU is an Assistant Professor in the Department of Systems Engineering and Operations Research at George Mason University. He received his Ph.D. from the Department of Industrial Engineering and Management Sciences of Northwestern University. His research interests include Monte Carlo simulation, stochastic optimization, computational intelligence, and applications in risk management and aviation. He is a member of INFORMS and IEEE and is a senior member of IIE. His email address is jxu13@gmu.edu and his web page is http://mason.gmu.edu/ jxu13/. 